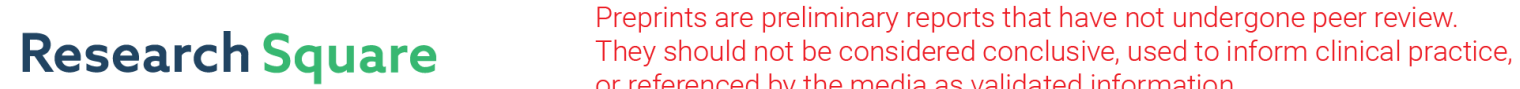 or referenced by the media as validated information. \\ Requirement for Containing Etoposide in the Initial Treatment of Lymphoma Associated Hemophagocytic Lymphohistiocytosis
}

\section{Yue Song}

Capital Medical University Affiliated Beijing Friendship Hospital

Jingshi Wang

Capital Medical University Affiliated Beijing Friendship Hospital

\section{Yini Wang}

Capital Medical University Affiliated Beijing Friendship Hospital

\section{Lin Wu}

Capital Medical University Affiliated Beijing Friendship Hospital

Zhao Wang ( $\square$ wangzhao@ccmu.edu.cn )

Capital Medical University Affiliated Beijing Friendship Hospital

\section{Research article}

Keywords: hemophagocytic lymphohistiocytosis, lymphoma, etoposide, treatment, prognosis, Epsteinbarr virus

Posted Date: July 10th, 2020

DOl: https://doi.org/10.21203/rs.3.rs-40791/v1

License: (c) (i) This work is licensed under a Creative Commons Attribution 4.0 International License. Read Full License 


\section{Abstract}

Background: Hemophagocytic lymphohistiocytosis $(\mathrm{HLH})$ is a severe or even fatal inflammatory status caused by a hereditary or acquired immunoregulatory abnormality. Lymphoma associated hemophagocytic lymphohistiocytosis (LAHS) is a kind of secondary HLH (sHLH). It has the worst prognosis among sHLH. However, there's still no standard treatment strategy. The argument mainly focuses on whether an HLH-directed or malignancy-directed approach should initially be adopted. Etoposide is one of the key drugs in HLH treatment, also effective in lymphomas. We sought to identify the importance of containing etoposide in initial treatment, comparing with the chemotherapy which directed at lymphoma but without etoposide.

Methods: 66 patients diagnosed as LAHS in our center between Jan 12015 and Dec 312017 were divided into two groups according to weather the initial treatment containing etoposide, or other lymphoma-directed chemotherapy without etoposide.

Results: The remission rate of the initial etoposide group (52 patients) is significantly better than that of no initial etoposide group ( 14 patients) $(73.1 \%$ vs. $42.9 \%, p=0.033)$. The relapse occurrence between two groups shows difference $(26.3 \%$ vs. $50 \%)$ but not significant $(p=0.339)$. There were 28 deaths in patients with initial etoposide (mortality rate 53.8\%) and 12 deaths in the without-initial-etoposide group (mortality rate $85.7 \%)$. A significant difference in mortality was noted between the two groups $(p=0.030)$. The $2-$ month survival $(79.8 \%$ vs. $46.8 \%, p=0.019)$ and overall survival (median survival time $25.8 \mathrm{w}$ vs. $7.6 \mathrm{w}, \mathrm{p}=$ 0.048 ) of the initial etoposide contained group is also better. Multivariate cox analysis revealed that for patients without EBV infection (37 cases), initial treatment with etoposide could significantly improve prognosis $(p=0.010, \operatorname{Exp}(B)=0.183)$, but for patients with positive $E B V$, it's not significant.

Conclusions: We concluded that containing etoposide is essential in the initial treatment of LAHS, weather using the HLH directed or lymphoma directed strategy. It provides higher response rate, lower mortality rate and better survival, especially for EBV negative patients.

\section{Background}

Hemophagocytic lymphohistiocytosis (HLH) is a severe or even fatal inflammatory status caused by a hereditary or acquired immunoregulatory abnormality [1, 2]. It is divided into two categories: primary and secondary. Secondary HLH (sHLH) is often associated with and caused by infections, malignant tumors, and autoimmune diseases. Malignancy HLH (M-HLH) is a kind of $\mathrm{sHLH}$, and lymphoma associated (LAHS) is the most common kind of M-HLH. M-HLH suffers the worst prognosis in secondary HLH[3], which has a high early mortality and the median survival is considered to be less than 2 months[4]. That is because that the treatment of LAHS is often faced with many difficulties, such as HLH controlling problem, the high recurrence rate, the lymphoma is hard to relieve, and so on. However, there's still no standard strategy for LAHS's treatment up until now. There are no universal conclusions on whether an HLH directed, malignancy-directed or combined approach should initially be adopted[5]. In the study of 
adult M-HLH by Gevorg Tamamyan in 2016, they noted a very aggressive and rapid progression of HLH with $\mathrm{M}-\mathrm{HLH}$, with a majority of patients dying from $\mathrm{HLH}$ within 2-4 weeks in spite of continued treatment of the underlying malignancy[4]. In the consensus review on malignancy-HLH in adults in 2017, it is suggested that once HLH-triggered organ damage occurred, the application of lympholytic agents must be considered, including etoposide, corticosteroids, polyvalent immunoglobulins which targets the cytokine storm and T-cell proliferation, and DEP regimen as salvage therapy. Neoplastic specific treatment should be put after the organ function is re-established[5]. However, it is noted only as an expert opinion, prospective, randomized or controlled clinical trials is still needed to support. Etoposide is one of the key drugs in HLH treatment[6], and its importance and necessity, especially in initial treatment of some types of $\mathrm{HLH}$, are getting a lot attention. In previous report, the initial treatment with etoposide is believed to improve the prognosis of EBV-HLH patients, especially in adult patients[7, 8]. In the Alison S. and Nancy B.'s experience of HLH treatment, they suggested that etoposide containing chemotherapy regimen in LAHS should be strongly considered[9]. Also, in 2018,Camille Bigenwald et al. reanalyzed 71 cases of LAHS and found out that etoposide contained is an independent factor in improving prognosis[10]. Beside of the controlling HLH cytokine storm, etoposide also has a certain anti-tumor effect, especially in lymphoma[11]. By comparing the etoposide-containing regimen and the lymphoma specialized chemotherapy without etoposide, sought to identify the importance of including etoposide in the initial treatment of LAHS. Can etoposide control HLH at an early stage and also play a certain antilymphoma effect, so that it may address the treatment of HLH and lymphoma at the same time? This may lay a foundation for the future better treatment strategy of LAHS.

\section{Methods}

\section{Patients}

In total, 66 patients diagnosed as LAHS of all ages admitted to our center between Jan 12015 and Dec 312017 were retrospectively reviewed. All patients met the HLH-04 diagnostic criteria[6] and pathology confirmed as lymphoma. The lymphoma was considered to be concomitant with the HLH when it was diagnosed within the two months of HLH diagnosis. Those LAHS caused by chemotherapy during lymphoma treatment were excluded.

\section{Methods}

These patients were divided into two groups according to the initial treatment regimen containing etoposide or not. The first group is that etoposide was administrated in the initial treatment, and the second group didn't contain etoposide in the initial treatment regimen, but with the lymphoma-directed chemotherapy.

The positive EBV is defined as EBV-DNA copy number of peripheral blood $\geq 500$ copies $/ \mathrm{ml}$ (pass the EBV international standard, namely: NIBSC number 09/260 for detection[12]), or EBV-encoded small RNA (EBER) + in tissues. 


\section{Evaluation criteria and observed indicators}

Age, gender, pathological type of lymphoma, IPI (International prognostic index) scores, initial clinical symptoms as well as laboratory findings, initial treatment regimen, response rate, transplantation, and outcome of each patient.

As this study is mainly focusing on the HLH part of LAHS, so the assessment of the efficacy of LAHS followed the criteria proposed by Marsh et al and revised by Yini Wang et al [13, 14]. A complete response (CR) was defined as the normalization of all quantifiable symptoms and laboratory markers of HLH, including levels of soluble CD25, ferritin, and triglyceride; hemoglobin levels; neutrophil and platelet counts; and alanine aminotransferase (ALT) levels. A partial response (PR) was defined as improvement in two or more of the following quantifiable symptoms and laboratory markers by 2 weeks: 1.5 -fold decrease in soluble CD25 response; ferritin and triglyceride decreases of at least $25 \%$; an increase of at least $100 \%$ to $>0.5^{\star} 10^{9} / \mathrm{L}$ in patients with an initial neutrophil count of $<0.5^{\star} 10^{9} / \mathrm{L}$; an increase of at least $100 \%$ to $>2.0 * 10^{9} / \mathrm{L}$ in patients with an initial neutrophil count of 0.5 to $2.0 * 10^{9} / \mathrm{L}$; and a decrease of at least $50 \%$ in patients with initial ALT levels $>400 \mathrm{U} / \mathrm{L}$. Additionally, the subject's body temperature must have reverted to normal ranges for either $\mathrm{CR}$ or PR to be diagnosed. Failure to achieve PR was defined as no response.

Besides the clinical manifestation mentioned above and laboratory findings for efficacy evaluation, considering the main side effects of etoposide and lymphoma-directed chemotherapy, the level of myelosuppression was observed and evaluated by the "2016WHO Classification criteria for acute and subacute toxic side effects of anticancer drugs".

\section{Survival time}

Survival times were calculated from the date of diagnosis of LAHS. All patients were followed up until death or 31 Dec 2018, whichever occurred first. Patients undergoing stem cell transplantation were censored on the date of that procedure.

\section{Statistical analysis}

SPSS 22.0 (IBM, New York/USA) statistical software was adopted, and data that did not fit a normal distribution are presented as median and range. T-test was used for data that fit a normal distribution and homogeneity of variance, and Wilcoxon rank sum test was used for others. Kaplan-Meier survival curves were used to analyze the patients' survival. Multi-factor analysis using regression model COX risk analysis and the factors included the age of onset, the gender of the patient, with/without EBV infection, the type of lymphomas (B or T/NK), the IPI scores of lymphomas, and weather the initial treatment contained etoposide. $\mathrm{P}<0.05$ was considered to denote a significant difference.

\section{Results}


In the 66 patients, 39 were male, and 27 were female. The male to female ratio was 1.4:1. The median age of the patients was 48 years (15-76 years). Only two patients were under 16 years old, and both of them were 15 years old. As for the type of lymphoma, 32 patients were B cell lymphomas (29 B-cell nonHodgkin lymphoma, 3 Hodgkin lymphoma) and the other 34 were T/NK cell lymphomas ( 18 NK/T cell lymphoma, 16 other T-cell non-Hodgkin lymphoma). 29 (43.9\%) patients were complicated with EBV infextion.

\section{Treatment and outcome}

According to the differences of initial treatment regimen, the patients were divided into two groups according to weather the initial treatment containing etoposide. There were 52 patients with initial etoposide and 14 without one. The baseline level between two group shows no differences $(p>0.05)$ (Table 1). The treatment regimens contained initial etoposide include HLH-94/04 regimen, DEP (doxorubicin-etoposide-methylprednisolone), L-DEP (PEG-aspargase and DEP regimen), E-CHOP (etoposide, cyclophosphamide, hydroxydaunorubicin, vincristine, prednisone) and RE-CHOP (Rituximab and $\mathrm{E}-\mathrm{CHOP}$ regimen); for those lymphoma-directed chemotherapy but without etoposide, the regimens include CHOP/COP, R-CHOP/COP, L-CHOP (PEG-aspargase and CHOP regimen), CVAD

(cyclophosphamide, vincristine, doxorubicin, and dexamethasone), L-GDP, and P-Gemox (PEG-aspargase, Gemcitabine, Oxaliplatin). The dose of etoposide is $75-100 \mathrm{mg} / \mathrm{m}^{2}$ for HLH-94/04 regimen, $100 \mathrm{mg} / \mathrm{m} 2$ for DEP/L-DEP regimen once a week[13, 15], 60-100 mg/m2 d1-d3 for E-CHOP regimen. 
Table 1

Initial clinical characteristics of the patients between 2 groups.

\begin{tabular}{|c|c|c|c|}
\hline Features & With etoposide & Without etoposide & p valve \\
\hline \multicolumn{4}{|l|}{ Age, years } \\
\hline Median & 48 & 44.5 & 0.881 \\
\hline Range & {$[15,76]$} & {$[29,65]$} & \\
\hline Gender & & & 0.544 \\
\hline Male (n) & 32 & 7 & \\
\hline Female (n) & 20 & 7 & \\
\hline Type of lymphoma & & & 0.635 \\
\hline B cell & 26 & 6 & \\
\hline T/NK cell & 26 & 8 & \\
\hline Fever $\left(\mathrm{T}>38.5^{\circ} \mathrm{C}\right)$ & 52 & 14 & \\
\hline Splenomegaly (n) & $38(73.1 \%)$ & $11(78.6 \%)$ & 1.00 \\
\hline Haemophagocytosis (n) & $45(86.5 \%)$ & $11(78.6 \%)$ & 0.431 \\
\hline WBC $\left(* 10^{9} / \mathrm{L}\right)$ & $2.25[0.06,17.00]$ & $3.25[0.4,8.75]$ & 0.165 \\
\hline $\mathrm{HGB}(\mathrm{g} / \mathrm{L})$ & $90.05 \pm 21.84$ & $81.71 \pm 15.11$ & 0.184 \\
\hline $\operatorname{PLT}\left({ }^{*} 10^{9} / \mathrm{L}\right)$ & $47.5[3,344]$ & $61.5[9,392]$ & 0.252 \\
\hline $\operatorname{ALT}(\mathrm{U} / \mathrm{L})$ & $52.5[7.0,798.0]$ & $41.5[13,235]$ & 0.753 \\
\hline AST (U/L) & $56.4[3.3,520.2]$ & $43.9[14.3,990.0]$ & 0.869 \\
\hline LDH & $556[137,4581]$ & $990[76,1986]$ & 0.569 \\
\hline Total bilirubin $(\mu \mathrm{mol} / \mathrm{L})$ & $24.77[6.29,348.15]$ & $20.70[6.50,213.58]$ & 0.718 \\
\hline ALB & $28.5[19.2,44.0]$ & $28.7[20.1,34.8]$ & 0.389 \\
\hline $\mathrm{Cr}$ (umol/L) & $59.7[35.5,125]$ & $64.4[37.7,272.3]$ & 0.725 \\
\hline $\mathrm{TG}(\mathrm{mmol} / \mathrm{L})$ & $2.45 \pm 1.22$ & $2.95 \pm 1.49$ & 0.215 \\
\hline $\mathrm{Fbg}(\mathrm{g} / \mathrm{L})$ & $1.76[0.16,8.47]$ & $3.7[0.73,8.68]$ & 0.017 \\
\hline Ferritin $(\mathrm{ng} / \mathrm{mL})$ & $2307.5[204.3,75000]$ & $2574[557.3,45487]$ & 0.734 \\
\hline $\mathrm{sCD} 25(\mathrm{pg} / \mathrm{mL})$ & $36605[661,44000]$ & $34749[3827,44000]$ & 0.903 \\
\hline
\end{tabular}

the valve was expressed as median [range] or mean \pm standard deviation. 


\begin{tabular}{|llll|}
\hline Features & With etoposide & Without etoposide & p valve \\
\hline IPI scores & $3.00 \pm 0.913$ & $3.29 \pm 0.994$ & 0.315 \\
\hline EBV-positive & $23(44.2 \%)$ & $6(42.8 \%)$ & 0.927 \\
\hline \multicolumn{2}{|l}{ the valve was expressed as median [range] or mean \pm standard deviation. } \\
\hline
\end{tabular}

The response rates of the 66 patients was $66.7 \%$ (44/66), with the CR rate of $24.2 \%(16 / 66)$ and PR rate of $42.4 \%(28 / 66)$. A total of 38 cases with initial etoposide achieved remission, and the remission rate was $73.1 \%$ (38/52), with the CR rate 28.8\% (15/52) and PR 44.2\% (23/52). 6 cases used chemotherapy without etoposide achieved remission, and the remission rate was $42.9 \%(6 / 14)$, with the CR rate $7.1 \%$ $(1 / 14)$ and PR rate $35.7 \%(5 / 14)$. A significant difference was noted between the two groups $(p=0.033)$. The level of bone marrow suppression shows no differences between two groups of patients $(p=0.122)$. In the 44 patients achieved CR/PR, there were 13 patients suffering HLH relapse. The relapse occurrence between two groups shows difference $(26.3 \%, 10 / 38 v s .50 \%, 3 / 6)$ but not significant $(p=0.339)$.

A total of 40 deaths occurred with a total mortality rate of $60.6 \%$, almost half of them occurred in the first 2 months $(42.5 \%, 17 / 40)$ and most of them occurred in the first 6 months after diagnosis $(77.5 \%, 31 / 40)$. There were 28 deaths in patients with initial etoposide (mortality rate 53.8\%) and 12 deaths in the without-initial-etoposide group (mortality rate $85.7 \%$ ). A significant difference in mortality was noted between the two groups $(p=0.030)$. For the cause of death, in the with-initial-etoposide group, there were 24 patients died of HLH progression $(85.7 \%, 24 / 28)$, and 4 died of complications. In the without-initialetoposide group, there were 11 patients died of $\mathrm{HLH}$ progression $(91.7 \%, 11 / 12)$, and 1 patient died during the conditioning before allo-HSCT.

\section{Survival}

The median survival of all patients is $23.8 \mathrm{w}[95 \% \mathrm{Cl}(15.5,32.2)]$. For the with-initial- etoposide group, the median survival is $25.8 \mathrm{w}$ [ $95 \% \mathrm{Cl}(13.2,38.5)]$, and for the without-initial-etoposide group, is $7.6 \mathrm{w}$ [ $95 \%$ (0, 19.8)]. The overall survival of the with-initial-etoposide group is significantly better than the other one $(p=$ 0.048) (Fig. 1). Considering almost half of patients died within 8 weeks ( 2 months), the overall survival at 2 months was estimated as $79.8 \%$ vs. $46.8 \%$, with a significant difference $(p=0.019)$. The treatment efficacy evaluation suggested that the overall survival was significantly prolonged in patients who reached CR/PR compared with patients who did not reached response $(p<0.01)$ (Fig. 2). Also, patients with positive EBV suffered a worse prognosis $(p=0.002)($ Fig. 3$)$.

Multivariate cox analysis revealed that: for patients with EBV infection (29 cases), the type of lymphoma (B or T/NK cell) $(p=0.011)$ (Fig. 4a) and the IPI score $(p=0.004)$ was associated with prognosis (Fig. $4 b)$, while whether etoposide was included in the initial treatment had no significant effect on prognosis $(P=$ 0.474). However, in patients without EBV infection (37 cases), multivariate cox analysis showed that initial treatment with etoposide was significantly related to prognosis $(p=0.010, \operatorname{Exp}(B)=0.183)$, while the type of lymphoma $(p=0.259)$ and IPI score $(p=0.419)$ didn't show significantly effects (Fig. 5$)$. 


\section{Discussion}

Hemophagocytic lymphohistiocytosis (HLH) is a severe or even fatal inflammatory status caused by a hereditary or acquired immunoregulatory abnormality, non-malignant proliferation of lymphocytes and tissue cells, and secretion of a large number of inflammatory cytokines[1, 2, 16]. Acquired HLH is often associated with and caused by infections, malignant tumors, and autoimmune diseases. Malignancy $\mathrm{HLH}$ is one of the most common secondary HLH, especially in adult patients. M-HLH may occur in up to $1 \%$ of patients with hematologic malignancies, and the lymphoma associated HLH (LAHS) is the most common type in M-HLH[17]. M-HLH is considered to suffer the worst outcome among the secondary $\mathrm{HLH}[3,18]$. The mortality of MAS (macrophage activation syndrome) secondary to sJIA (systemic juvenile idiopathic arthritis) is only $8 \%$, while the mortality rate of $\mathrm{M}-\mathrm{HLH}$ is even $>80 \%[3]$. The median survival time of M-HLH is only 2 months $[4,5,18]$. However, the standard treatment strategy is still controversial. First, HLH-94/04 regimen is based on the study of patients $<16$ years old, but actually, most of LAHS patients are adults[19]. Only 2 patients with LAHS in this study were $<16$ years old. Previous reports have also confirmed that the prognosis of adult HLH is worse than that of children[20]. HLH-94 is not suitable enough for LAHS. Second, the priority of HLH-directed treatment and lymphoma-directed treatment is still controversial[4, 5, 21], even though in some clinical observation, many patients died of $\mathrm{HLH}$ within 2-4 weeks in spite of continued treatment of the underlying malignancy[5]. Third, due to the severe cytopenia caused by HLH, cytotoxic therapy's usage, such as etoposide, is limited for the concern of myelosuppression. The above three reasons lead to LAHS's urgent need for relevant clinical study to play a certain therapeutic guiding significance. Etoposide is one of the core drugs in the $\mathrm{HLH}-94 / 04$ standard protocol, and its importance in the treatment of HLH has been confirmed, such as the improvement of prognosis in the initial treatment of EBV-HLH$[7,8,20]$. But its significance in LAHS treatment has not yet been discussed. In the "How I treat" article in adult HLH, Alison S. and Nancy B. reported one case of LAHS patient who was effectively treated with etoposide combined with CHOP regimen[9]. Camille Bigenwald et al. re-evaluated 162 patients with secondary HLH reported by Rivière $S$ in 2014 in France[10]. Reanalysis of 71 patients with LAHS data revealed that etoposide was an independent prognostic factor in the treatment of HLH. In the 2017 adult M-HLH expert consensus review, it is recommended to prioritize treatment for cytokine storms and T cell proliferation, such as etoposide, for patients with HLH who have experienced severe organ damages[5]. In this study, it was evident that the early response rate, short-term survival, and overall survival of the treatment group containing etoposide were superior to those of lymphoma directed chemotherapy without etoposide. Even though we cannot draw a conclusion about the priority between HLH or lymphoma directed treatment, it can be concluded that no matter which method is chosen, the inclusion of etoposide in initial therapy, accounts for a definitely better prognosis.

The mechanism of etoposide in HLH is that it reduces the production of excessive proinflammatory factors by selectively ablating over-activated $T$ cells and inhibiting mononuclear-macrophage activation[22]. This effect is different from immunosuppressive effects of corticosteroids and the immunomodulatory effects of IVIG or cyclosporin, which makes it indispensable in some kind of HLH treatment strategy. In some previous studies, the median survival time of M-HLH is only 8 weeks $[4,18]$. In 
the study of Camille B et al, by observing the outcome of B-NHL and T-NHL-related HLH, they have an opinion that the long-term lymphoma prognosis may be comparable to a similar lymphoma not associated with HLH, once the initial and crucial early period is overcome[10]. And, thus, the long-term prognosis improvement of lymphoma associated with HLH may be accomplished through the optimization of management during the initial HLH diagnostic period. In our study, it was observed that nearly half of all deaths occurred within 2 months after diagnosis, and the median survival of the withoutetoposide group was only 7.8 weeks. In addition to the early response rate, the improvement of 2-month survival rate was also significant in etoposide-contained group, and we have reason to believe that the improvement in short-term survival is also why the etoposide group contributes to a better long-term survival. The poor prognosis of LAHS has be considered relating to its pathogenesis, that the lymphoma may drive secondary HLH by its ability to secrete inflammatory cytokines, or to present antigens that can activate a cytotoxic $T$ cell response. The underlining malignancy plays a crucial role in the development of HLH. Etoposide is a chemotherapy medication used for the treatment of a number of cancers, including lymphoma. It forms a ternary complex with DNA and the topoisomerase II enzyme (that aids DNA unwinding), preventing re-ligation of the DNA strands. Thus, etoposide causes DNA strands to break[23]. Although etoposide is not currently a commonly used first-line regimen in lymphoma, it has been reported that it can improve EFS and PFS in some patients with T-cell lymphoma under 60 years old[11, 24], also used in some B-cell lymphoma[25], indicating that the usage of etoposide in LAHS, not only effects on controlling $\mathrm{HLH}$, but also may affect a little on the underlining lymphoma. In addition, although the relapse rate in the containing etoposide group was lower than that in the etoposide-free group, the difference was not statistically significant and may be related to the limited number of data. The persistence of etoposide's effect, treatment course and dosage still need further clinical data.

For the intervention time of etoposide, considering the high early mortality observed in this study, and that the prognosis of LAHS patients who achieved early remission was significantly better than those failed to achieve, and that the inclusion of etoposide is a significant improvement in early remission rate, therefore, it is appropriate to include etoposide in the initial treatment. And as the lymphoma directed treatment requirement for subsequent therapy, early effective control of inflammatory conditions provides good conditions for subsequent therapy and even allo-HSCT. However, the sever cytopenia caused by HLH may bring some concern for the usage of etoposide. One of the main reasons why less than $50 \%$ of LAHS patients received HLH directed treatment, is the concern of myelosuppression deterioration after cytotoxic therapy. But in fact, etoposide's efficiency occurs within 24-48 $\mathrm{h}$ and in $\mathrm{HLH}$, far outweighs the risk of transient worsening of the cytopenia[6,20]. Previous reports are also considered to be in the treatment of $\mathrm{HLH}$, and in some circumstances, etoposide prevented the development of cytopenia and marrow hypocellularity[26]. This study evaluated the level of myelosuppression and there was no significant difference between the two groups, but the etoposide dose in this study was indeed adjusted according to adult intolerance and taking into account the cytopenia. It seems that as long as appropriate doses, concerns about myelosuppression are not sufficient to constitute a good reason for not using etoposide. On the other side, there are truly 4 deaths of complications in the etoposide group. Therefore, after the 
reduction of etoposide, whether its anti-tumor and anti-HLH effects are affected, and whether the risk of serious infection and bleeding complications increases, further research is needed.

It is interesting that in the multivariate prognosis analysis, it was found that in patients with positive EBV, whether etoposide was included in the initial treatment had little effect on the prognosis, but in patients who did not suffer EBV infection, the initial treatment included etoposide could clearly improve the prognosis. In previous reports, whether it is T / NK-cell or B-cell lymphoma, the presence of EBV infection is a clear poor prognostic factor. The meta-analysis in 2018 of 356 patients suggested that, the pretreatment positive EBV was significantly correlated with the overall survival of extra-nodal NK/T-cell lymphoma (pooled $H R=3.78, p=0.004$ )[27]. EBV-positive diffuse large B-cell lymphoma also contribute a poor prognosis[28]. Also, as we all know, the type of lymphoma secondary to HLH is closely related to the prognosis. In the study of Sano $\mathrm{H}$ et al., the rate of early death was higher in patients with T/NK-cell LAHS than in those with B-cell LAHS (62.5 vs. 10.5\%)[29]. However, in this study, for patients without positive EBV, the type of lymphoma was not significantly correlated with prognosis. This result suggests that the worse prognosis of T/NK-cell LAHS may be related with that EBV infection is more common in T/NK cell lymphoma. EBV itself can cause HLH, and whether it is EBV-HLH or EBV (+) NK/T-LAHS[3, 30], combined with positive EBV is an independent prognostic factor, as also observed in our study (Fig. 3). Therefore, a possible conjecture is that when lymphoma is secondary to HLH and combined with positive EBV, EBV influences the prognosis from both aspects of lymphoma and HLH. In this EBV + LAHS, the inclusion of etoposide in the initial treatment is not sufficient to improve the outcome. But for EBV- LAHS, initial treatment with etoposide is clearly helpful for prognosis and is certainly recommended.

\section{Conclusion}

As one of the most common secondary HLHs, LAHS suffers the worst outcome and most of patients died within a short term after diagnosis. However, no clear and unified initial treatment plan is currently available, and the debate about the priority of HLH-directed or lymphoma-directed is still going on. This study found that containing etoposide in the initial treatment plan significantly improve the early response rate, 2-month survival and overall survival, and especially prominent in EBV negative patients. Also, the level of bone marrow suppression shows no significant differences weather etoposide is contained. So, containing etoposide is essential in the initial treatment of LAHS, weather using the HLH directed or lymphoma directed strategy, especially for EBV negative patients. However, the relapse is still of concern and the most suitable LAHS therapy still needs more studying.

\section{Abbreviations}

Allo-HSCT: Allogeneic hematopoietic stem cell transplantation; WBC: White blood cells; HGB hemoglobin; PLT: Platelets; LDH: lactate dehydrogenase; ALB: albumin; Cr: creatinine; TG: Triglycerides; ALT: Alanine aminotransferase; CR: Complete response; DEP regimen: Doxorubicin hydrochloride liposome, etoposide, and methylprednisolone; EBER: EBV encoded small RNA; EBV: Epstein-Barr virus; HLH: Hemophagocytic 
lymphohistiocytosis; L-DEP regimen: PEG-aspargase plus DEP regimen; NK: Natural killer; OS: Overall survival; PR: Partial response; VP-16: Etoposide;.

\section{Declarations}

\section{Ethics approval and consent to participate}

Ethical approval: All procedures performed in studies involving human participants were in accordance with the ethical standards of the institutional and/or national research committee and with the 1964 Helsinki declaration and its later amendments or comparable ethical standards. The study was approved by the Ethics Committee at Beijing Friendship Hospital.

Informed consent: Written informed consent was obtained from all individual participants or guardian for participants under 16 years oldincluded in the study.

\section{Consent to publish}

\section{Availability of data and materials}

The datasets used during the current study are available from the corresponding author on request.

\section{Competing interests}

The authors declare that they have no competing Interest.

\section{Funding}

This work was supported by the National Natural Science Foundation of China (No.81871633); Beijing Natural Science Foundation (No.7181003); Beijing Municipal Administration of Hospitals' Ascent Plan (DFL20180101); Beijing Municipal Administration of Hospitals Incubating Program (PX2018003); Beijing Municipal Administration of Hospitals' Youth Program (QML20160102); and Beijing Municipal Administration of Hospitals Clinical Technology Innovation Project (XMLX201803). The funding body did not contribute to the design of the study and collection, analysis, and interpretation of data, or in writing the manuscript.

\section{Authors' contributions}

ZW contributed to the design of the study. YNW, JSW and LW helped with the study design and data analyses. YS conducted the data analysis and wrote the manuscript. All authors approved the final manuscript.

\section{Acknowledgements}

We thank the patients and their families for participating in our study. 


\section{References}

1. Henter JI, Samuelsson-Horne A, Arico M, Egeler RM, Elinder G, Filipovich AH, Gadner H, Imashuku S, Komp D, Ladisch S et al: Treatment of hemophagocytic lymphohistiocytosis with HLH-94 immunochemotherapy and bone marrow transplantation. Blood 2002, 100(7):2367-2373.

2. Arico M, Janka G, Fischer A, Henter JI, Blanche S, Elinder G, Martinetti M, Rusca MP: Hemophagocytic lymphohistiocytosis. Report of 122 children from the International Registry. FHL Study Group of the Histiocyte Society. Leukemia 1996, 10(2):197-203.

3. Otrock ZK, Eby CS: Clinical characteristics, prognostic factors, and outcomes of adult patients with hemophagocytic lymphohistiocytosis. American journal of hematology 2015, 90(3):220-224.

4. Tamamyan GN, Kantarjian HM, Ning J, Jain P, Sasaki K, McClain KL, Allen CE, Pierce SA, Cortes JE, Ravandi $\mathrm{F}$ et al: Malignancy-associated hemophagocytic lymphohistiocytosis in adults: Relation to hemophagocytosis, characteristics, and outcomes. Cancer 2016, 122(18):2857-2866.

5. Daver N, McClain K, Allen CE, Parikh SA, Otrock Z, Rojas-Hernandez C, Blechacz B, Wang S, Minkov $\mathrm{M}$, Jordan $\mathrm{MB}$ et al: A consensus review on malignancy-associated hemophagocytic lymphohistiocytosis in adults. Cancer 2017, 123(17):3229-3240.

6. Henter JI, Horne A, Arico M, Egeler RM, Filipovich AH, Imashuku S, Ladisch S, McClain K, Webb D, Winiarski $J$ et al: HLH-2004: Diagnostic and therapeutic guidelines for hemophagocytic lymphohistiocytosis. Pediatric blood \& cancer 2007, 48(2):124-131.

7. Song $Y$, Wang Y, Wang Z: Requirement for etoposide in the initial treatment of Epstein-Barr virusassociated haemophagocytic lymphohistiocytosis. British journal of haematology 2019, 186(5):717723.

8. Imashuku S, Kuriyama K, Teramura T, Ishii E, Kinugawa N, Kato M, Sako M, Hibi S: Requirement for etoposide in the treatment of Epstein-Barr virus-associated hemophagocytic lymphohistiocytosis. Journal of clinical oncology : official journal of the American Society of Clinical Oncology 2001, 19(10):2665-2673.

9. Schram AM, Berliner N: How I treat hemophagocytic lymphohistiocytosis in the adult patient. Blood 2015, 125(19):2908-2914.

10. Bigenwald C, Fardet L, Coppo P, Meignin V, Lazure T, Fabiani B, Kohn M, Oksenhendler E, Boutboul D, Uzzan $\mathrm{M}$ et al: A comprehensive analysis of Lymphoma-associated haemophagocytic syndrome in a large French multicentre cohort detects some clues to improve prognosis. British journal of haematology 2018, 183(1):68-75.

11. Schmitz N, Trumper L, Ziepert M, Nickelsen M, Ho AD, Metzner B, Peter N, Loeffler M, Rosenwald A, Pfreundschuh M: Treatment and prognosis of mature T-cell and NK-cell lymphoma: an analysis of patients with T-cell lymphoma treated in studies of the German High-Grade Non-Hodgkin Lymphoma Study Group. Blood 2010, 116(18):3418-3425.

12. Collaborative Study to Evaluate the Proposed 1st WHO International. 
13. Wang Y, Huang W, Hu L, Cen X, Li L, Wang J, Shen J, Wei N, Wang Z: Multicenter study of combination DEP regimen as a salvage therapy for adult refractory hemophagocytic lymphohistiocytosis. Blood 2015, 126(19):2186-2192.

14. Marsh RA, Allen CE, McClain KL, Weinstein JL, Kanter J, Skiles J, Lee ND, Khan SP, Lawrence J, Mo JQ et al: Salvage therapy of refractory hemophagocytic lymphohistiocytosis with alemtuzumab. Pediatric blood \& cancer 2013, 60(1):101-109.

15. Wang J, Wang $Y$, Wu L, Zhang J, Lai W, Wang Z: PEG-aspargase and DEP regimen combination therapy for refractory Epstein-Barr virus-associated hemophagocytic lymphohistiocytosis. Journal of hematology \& oncology 2016, 9(1):84.

16. Jordan MB, Allen CE, Weitzman S, Filipovich AH, McClain KL: How I treat hemophagocytic lymphohistiocytosis. Blood 2011, 118(15):4041-4052.

17. Machaczka M, Vaktnas J, Klimkowska M, Hagglund H: Malignancy-associated hemophagocytic lymphohistiocytosis in adults: a retrospective population-based analysis from a single center. Leukemia \& lymphoma 2011, 52(4):613-619.

18. Parikh SA, Kapoor P, Letendre L, Kumar S, Wolanskyj AP: Prognostic factors and outcomes of adults with hemophagocytic lymphohistiocytosis. Mayo Clinic proceedings 2014, 89(4):484-492.

19. Riviere S, Galicier L, Coppo P, Marzac C, Aumont C, Lambotte O, Fardet L: Reactive hemophagocytic syndrome in adults: a retrospective analysis of 162 patients. The American journal of medicine 2014, 127(11):1118-1125.

20. Arca M, Fardet L, Galicier L, Riviere S, Marzac C, Aumont C, Lambotte O, Coppo P: Prognostic factors of early death in a cohort of 162 adult haemophagocytic syndrome: impact of triggering disease and early treatment with etoposide. British journal of haematology 2015, 168(1):63-68.

21. Lehmberg K, Sprekels B, Nichols KE, Woessmann W, Muller I, Suttorp M, Bernig T, Beutel K, Bode SF, Kentouche $\mathrm{K}$ et al: Malignancy-associated haemophagocytic lymphohistiocytosis in children and adolescents. British journal of haematology 2015, 170(4):539-549.

22. Johnson TS, Terrell CE, Millen SH, Katz JD, Hildeman DA, Jordan MB: Etoposide selectively ablates activated $\mathrm{T}$ cells to control the immunoregulatory disorder hemophagocytic lymphohistiocytosis. Journal of immunology 2014, 192(1):84-91.

23. Pommier $\mathrm{Y}$, Leo $\mathrm{E}$, Zhang $\mathrm{H}$, Marchand $\mathrm{C}$ : DNA topoisomerases and their poisoning by anticancer and antibacterial drugs. Chemistry \& biology 2010, 17(5):421-433.

24. Ellin F, Landstrom J, Jerkeman M, Relander T: Real-world data on prognostic factors and treatment in peripheral T-cell lymphomas: a study from the Swedish Lymphoma Registry. Blood 2014, 124(10):1570-1577.

25. Dodero A, Guidetti A, Tucci A, Barretta F, Novo M, Devizzi L, Re A, Passi A, Pellegrinelli A, Pruneri G et al: Dose-adjusted EPOCH plus rituximab improves the clinical outcome of young patients affected by double expressor diffuse large B-cell lymphoma. Leukemia 2019, 33(4):1047-1051.

26. Mizrahi M, Ben-Chetrit E: Relapsing macrophage activating syndrome in a 15-year-old girl with Still's disease: a case report. Journal of medical case reports 2009, 3:138. 
27. Fei Q, Tian XK, Wu J, Zhu HM, Wang Y, Peng FY, Zhang WJ, Yin L, He X: Prognostic significance of Epstein-Barr virus DNA in NK/T-cell lymphoma: a meta-analysis. OncoTargets and therapy 2018, 11:997-1004.

28. Castillo JJ, Beltran BE, Miranda RN, Young KH, Chavez JC, Sotomayor EM: EBV-positive diffuse large B-cell lymphoma of the elderly: 2016 update on diagnosis, risk-stratification, and management. American journal of hematology 2016, 91(5):529-537.

29. Sano H, Kobayashi R, Tanaka J, Hashino S, Ota S, Torimoto Y, Kakinoki Y, Yamamoto S, Kurosawa M, Hatakeyama $\mathrm{N}$ et al: Risk factor analysis of non-Hodgkin lymphoma-associated haemophagocytic syndromes: a multicentre study. British journal of haematology 2014, 165(6):786-792.

30. Jin Z, Wang Y, Wang J, Wu L, Pei R, Lai W, Wang Z: Multivariate analysis of prognosis for patients with natural killer/T cell lymphoma-associated hemophagocytic lymphohistiocytosis. Hematology 2018, 23(4):228-234.

\section{Figures}

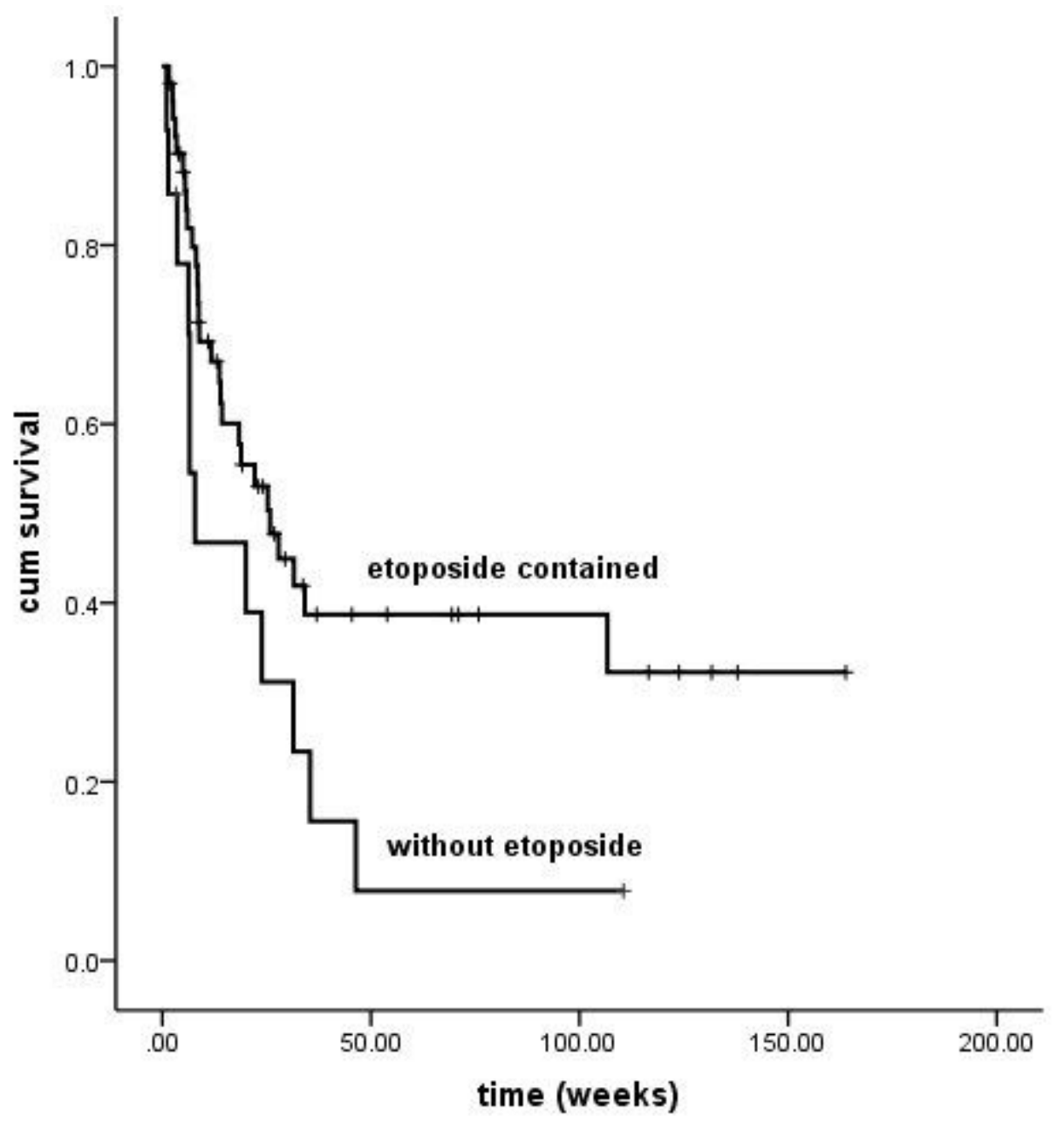

Figure 1 
Etoposide contained in initial treatment significantly improve the prognosis of LAHS, comparing with the only lymphoma directed therapy one $(p=0.048)$.

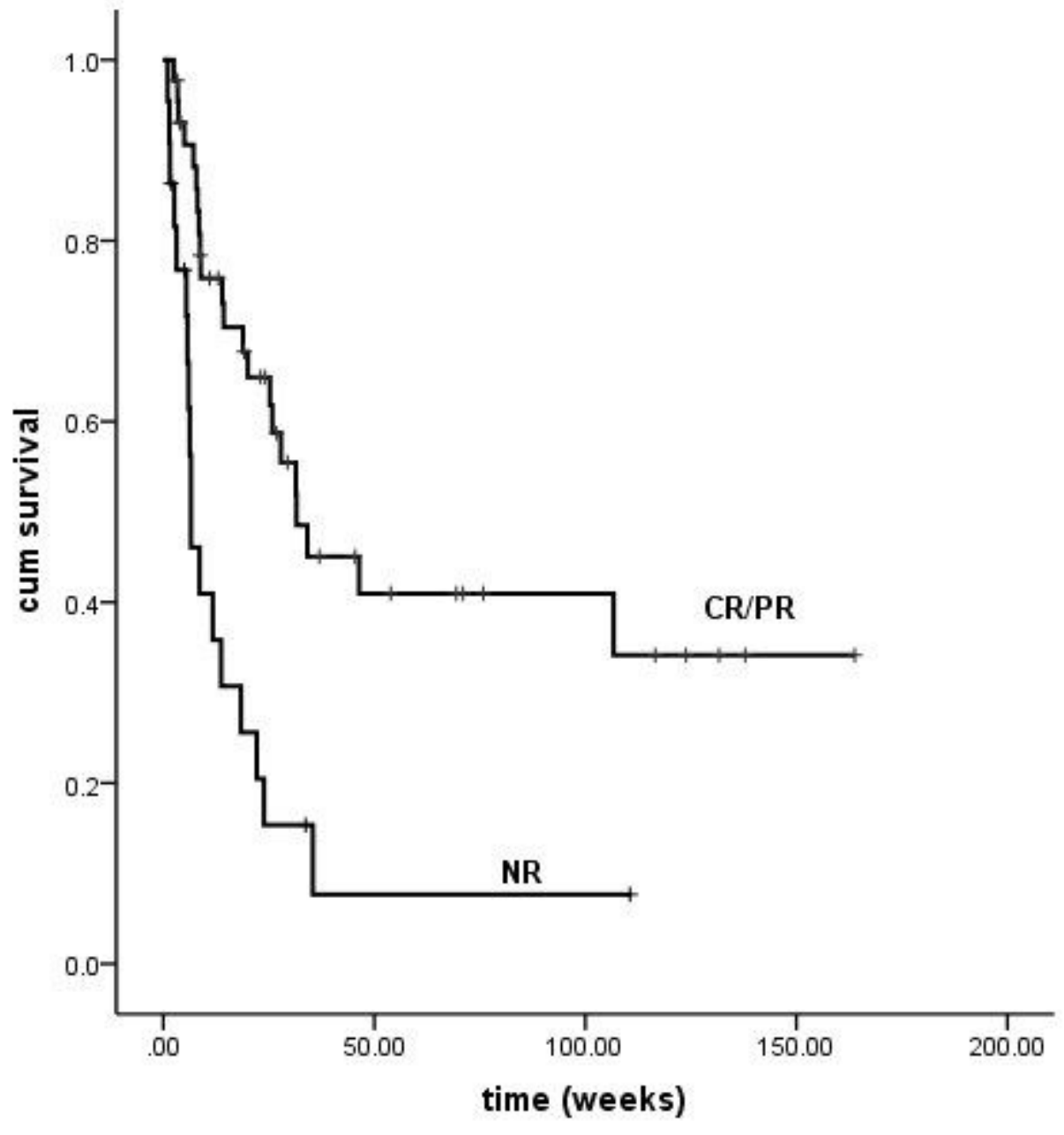

Figure 2

The treatment efficacy evaluation suggested that the overall survival was significantly prolonged in patients who reached CR/PR compared with patients who did not reached response $(p<0.01)$. 


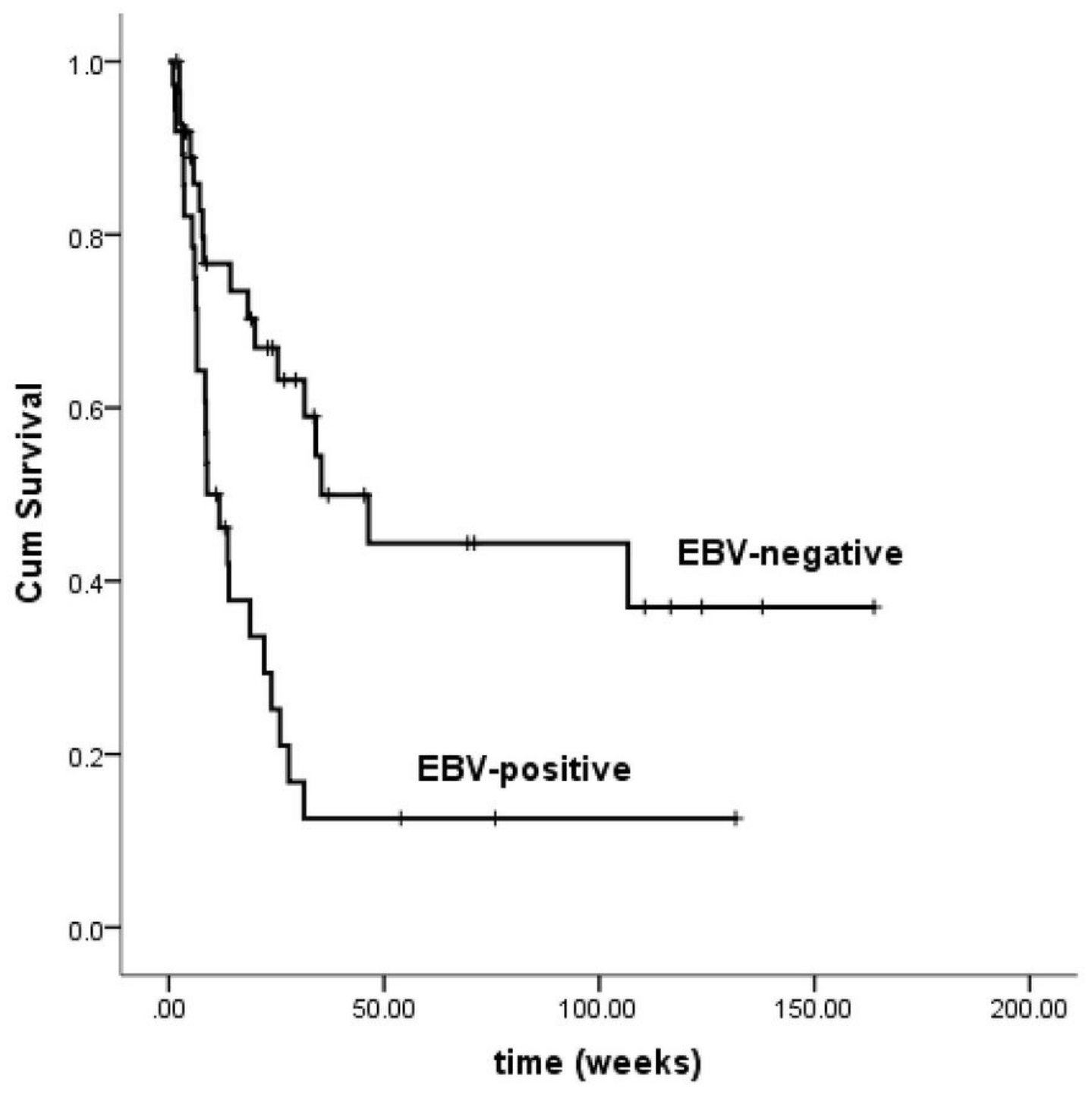

Figure 3

Patients with positive EBV suffered a worse prognosis $(p=0.002)$. 

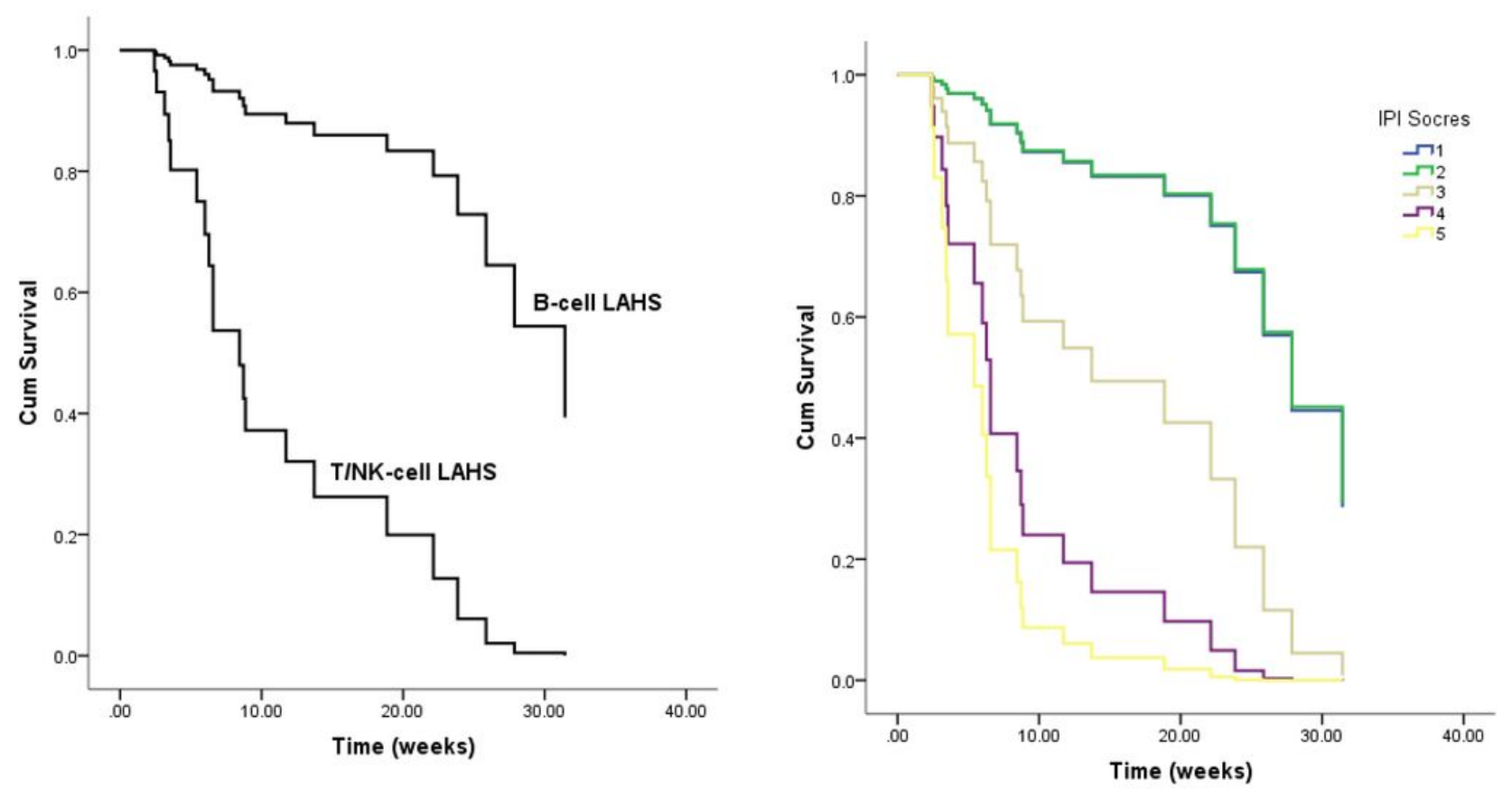

Figure 4

For EBV-positive patients, COX analysis: age of onset, the gender of the patient, the type of lymphomas (B or $\mathrm{T} / \mathrm{NK})$, the IPI scores of lymphomas, and weather the initial treatment contained etoposide, the results showed type of lymphoma (Figure 4a) $(P=0.011$, ExpB=0.113) and the IPI socores (Figure 4b) $(p=0.004$, $\operatorname{ExpB}=2.419)$ was associated with prognosis. 


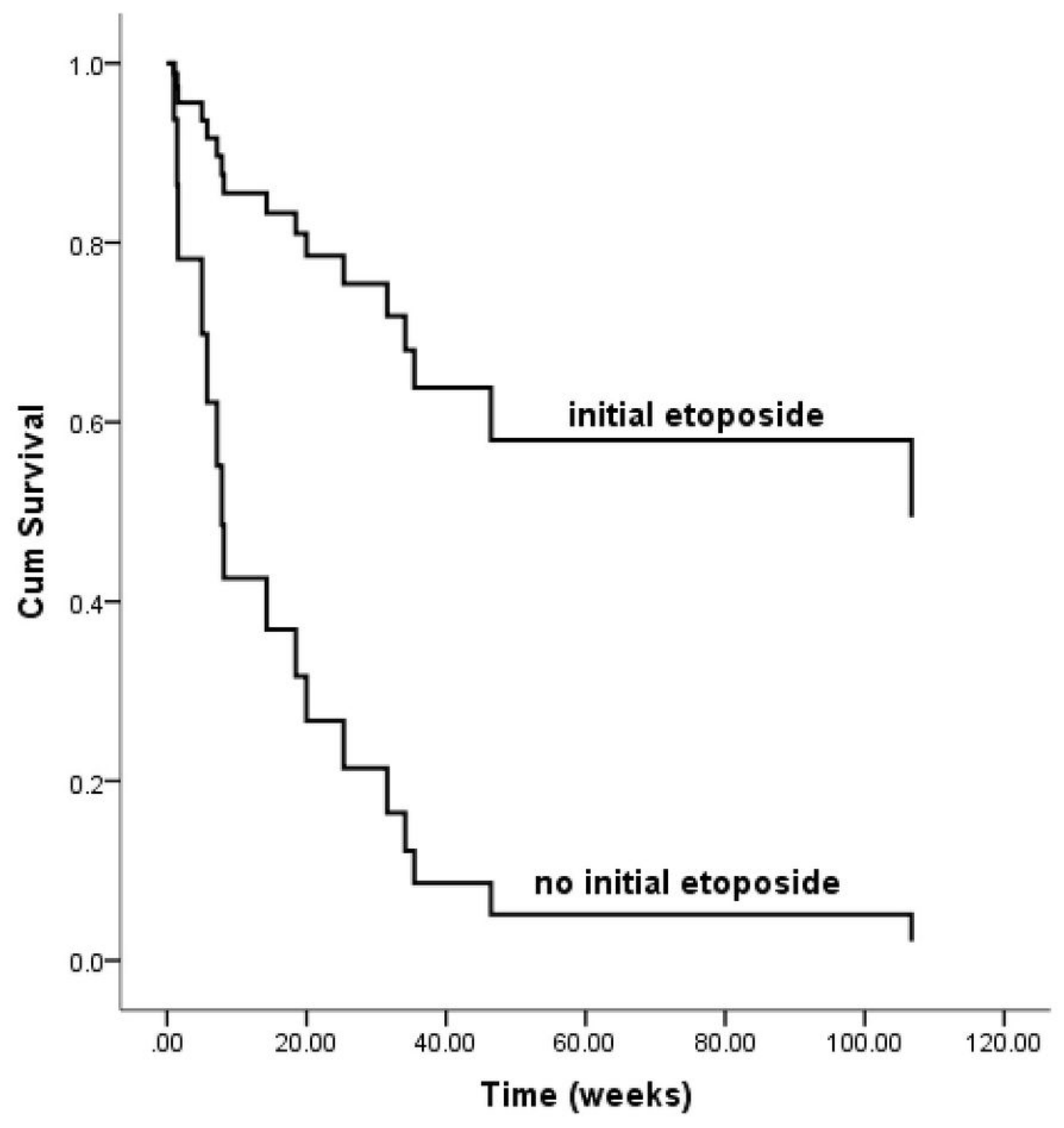

Figure 5

For EBV-negative patients, COX analysis: age of onset, the gender of the patient, the type of lymphomas (B or T/NK), the IPI scores of lymphomas, and weather the initial treatment contained etoposide, the results showed that weather initial treatment contained etoposide $(p=0.010, \operatorname{ExpB}=0.183)$ was associated with prognosis. 